\title{
A New System for Online Quantitative Evaluation of Optical See-Through Augmentation
}

\author{
Erin McGarrity Yakup Genc $^{\dagger}$ Mihran Tuceryan ${ }^{\ddagger}$ Charles Owen Nassir Navab $^{\dagger}$ \\ ${ }^{\dagger}$ Siemens Corporate Research \\ Imaging and Visualization Department \\ Princeton, NJ 08540, USA \\ ${ }^{\bullet}$ Computer Science \& Engineering Dept. \\ Michigan State University \\ East Lansing, MI 48824, USA \\ ${ }^{\ddagger}$ Dept. of Computer and Information Sci. \\ Indiana U. Purdue U. (IUPUI) \\ Indianapolis, IN 46202, USA
}

\begin{abstract}
A crucial aspect in the implementation of an augmented reality $(A R)$ system is determining its accuracy. The accuracy of a system determines the applications it can be used for. The aim of our research is measuring the overall accuracy of an arbitrary AR system. Once measurements of a system are made, they can be analyzed for determining the structure and sources of errors. From the analysis it may also be possible to improve the methods used to calibrate and register the virtual to the real.

This paper describes an online system for measuring the registration accuracy of optical see-through augmentation. By online, we mean that the user can measure the registration error they are experiencing while they are using the system. We overcome the difficulty of not having retinal access by having the user indicate the projection of a perceived object on a planar measurement device. Our method provides information which can be used to analyze the structure of the system error in two or three dimensions. The results of the application of our method to two monocular optical see-through AR systems are shown.
\end{abstract}

\section{Introduction}

A particular problem associated with calibrating optical see-through AR systems is determining the limits of accuracy. Since there is no way to access the augmented image on the user's retina, it is not possible to use traditional image-based measurement methods to determine system accuracy. Thus far, there have been two main approaches to accuracy verification for optical see-through displays. The first is to have the user calibrate the HMD and then report the qualitative accuracy of the alignment of a computer model to its real-world counterpart $[6,14,29]$, i.e., "acceptable," or "unacceptable." The second method uses a camera in place of the human eye and conducts image-based measurements to assess the accuracy of the alignment $[11,23]$. The former method is quick and easy, but it is subjective and gives no metric information. The latter method is performed by mounting a camera to the HMD so that it "sees" through the display panel. This is not very practical, nor does it guarantee that the measured accuracy will be observed by the user since a camera is only an approximation of the eye. Furthermore, the method is very tedious and must be performed offline since the camera is mounted where the user's head would normally be.

This paper presents a general system to quantitatively evaluate an optical see-through augmented reality system. Our system differs from previous systems in three ways. First, it allows a user to measure the error of the system while it is running. Second, it produces quantitative measurement of the error in registration of the real and the virtual. Finally, this error estimate includes errors injected by the user. With our system it is possible to estimate the accuracy limits of an application and detect structured errors in an optical see-through AR system.

The remainder of this paper is divided into 6 sections. Section 2 outlines previous work related to the topics of calibration and evaluation. Section 3 describes two AR systems used for testing the evaluation method. Section 
4 summarizes the calibration requirements and procedures for the components of both systems. Section 5 discusses the design requirements for an online evaluation system for optical see-through AR systems. In Section 5.1 a system and technique for evaluation are given, based on these requirements. The remainder of Section 5 gives experimental results for the application of the evaluation method to two different AR systems. These results are analyzed in two and three dimensions. Section 6 discusses the experimental results and their implications. Section 7 summarizes the results of the work.

\section{Previous Work}

There are several general works which describe AR as a whole. Azuma classifies AR systems based on display types and levels of immersion [1]. Feiner, et al., and Kutulakos and Vallino discuss workspace object representations in AR systems [6,18]. Klinker, et al., describe the merging of graphics and computer vision [17]. Fuchs and Rolland compare and contrast optical and video seethrough HMDs [7].

AR systems can be broadly categorized by the type of display they use. The first class is known as video see-through. Video see-through systems use a camera and display as an intermediary between the user and the scene. The video stream is captured by a camera, augmented and passed on to the user via a HMD or monitor. Much work has been done in this area. Tuceryan, et al., describe the calibration requirements for a video based AR system [28]. Janin, Mizell and Caudell developed a monocular system for assembling aircraft wiring harnesses [14]. Kato and Billinghurst describe a combined camera/HMD calibration scheme for tracking and augmenting fiducial markers in a scene [15]. Neumann and Cho present a method for improving the registration of such a system [25]. Mellor describes a procedure to calibrate a camera with embedded intrinsic parameters [24]. Other researchers have been concentrating on registration of 3D medical data to surgical patients $[3,4,9,19,30]$.

The second class of AR systems are called optical see-through systems. In optical see-through systems, the graphics are overlayed directly onto the user's view of the real world (via a half-silvered mirror or scanning laser.) Tuceryan and Navab present a user-friendly calibration method for optical see-through HMDs [29]. Oisho and Tachi discuss the calibration of a rigidly mounted optical see-through display [26]. MacIntyre and Coelho describe a method for adapting to the level of tracking error for AR systems [20]. Azuma and Holloway analyze sources of registration and tracking errors in AR systems [2,11,12]. This paper takes a similar di-

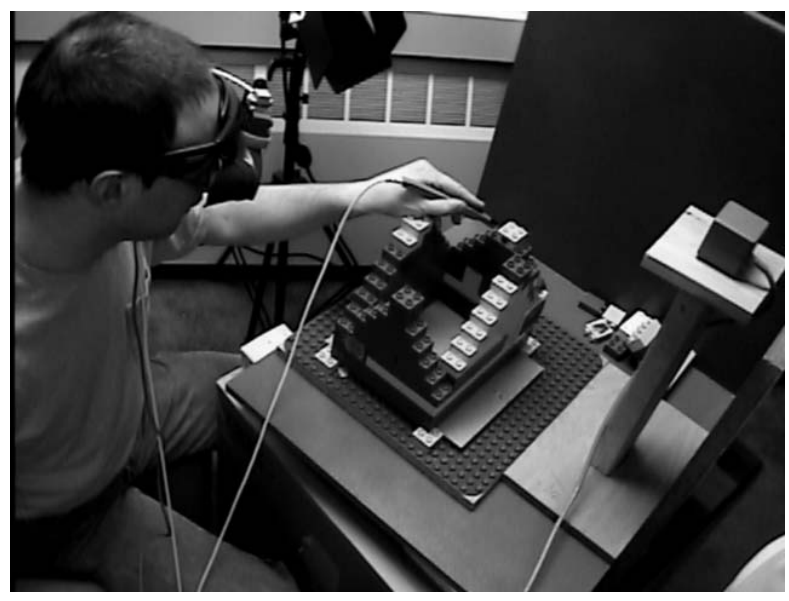

Figure 1. A photograph of the magnetically tracked system.

rection, but also provides an online method and apparatus for computing registration error. Previous work by our group in the realm of optical see-through calibration and validation can be found $[8,22,23]$.

\section{AR System Descriptions}

Two different optical see-through AR systems were tested for the experiments reported in this paper. The first system is based on a magnetic tracker. The second system uses an infrared video-based tracker. Both systems have stereo capable HMDs, but all experiments were performed in monocular mode. Details for the magnetically tracked system are given in Section 3.1. An overview of the system which uses video-based tracking is given in Section 3.2.

\subsection{AR System with Magnetic Tracker}

The first system consists of a SGI O2 workstation, a HMD and a magnetic tracker. A photograph of the system is shown in Figure 1. Figure 2 depicts a high level view of the setup.

The HMD for this system is a Sony Glastron LD100B. It has a resolution of $800 \times 600$ pixels per eye with a refresh rate of $60 \mathrm{~Hz}$. The stereo mode is framesequential for this display.

The magnetic tracker is a Polhemus Isotrak II with a single transmitter and two receivers: a marker and a digitizing stylus. It is capable of sensing the position and orientation of the receivers at ranges of up to one meter. The marker is rigidly attached to the HMD to 


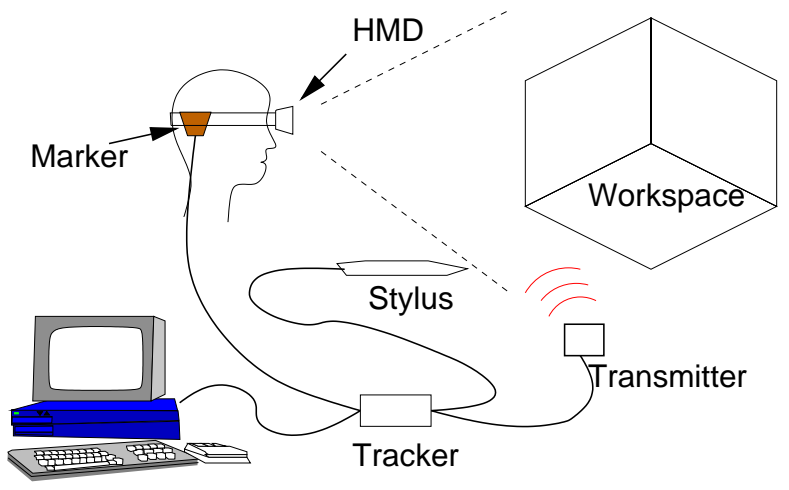

Figure 2. Magnetically tracked AR system.

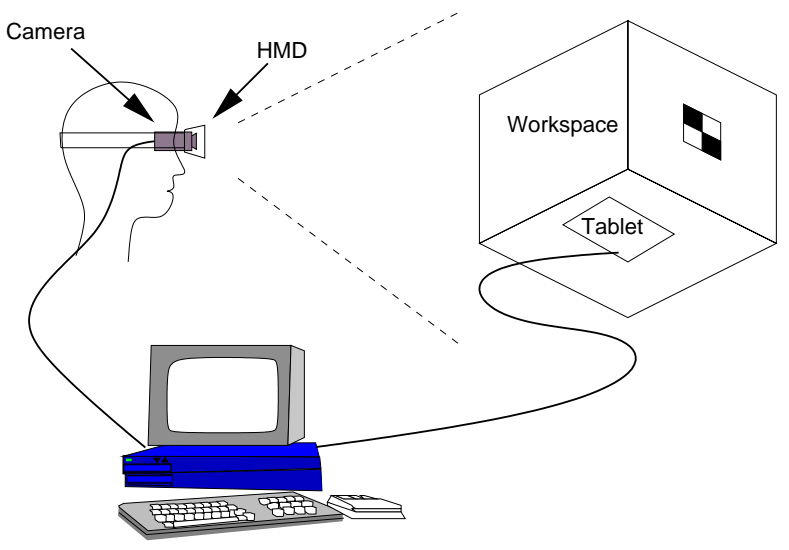

Figure 3. AR system with infrared videobased tracking. The tablet is used to perform the evaluation of the system.

track the user's head motion during runtime. The stylus can be used to measure the coordinates of real objects in the workspace at runtime. It can also be used to interact with virtual objects in the workspace.

\subsection{AR System with Video-Based Tracker}

The second optical see-through system is illustrated in Figure 3. The HMD for this system is a Virtual IO $i$-glasses ${ }^{\mathrm{TM}}$ field-sequential stereo display. It has a stereo resolution of $640 \times 240$ pixels per eye with a refresh rate of $60 \mathrm{~Hz}$. The monocular resolution for this display is $640 \times 480$. An infra-red vision-based tracker provides the system with position and orientation of the tracker camera attached to the HMD. The tracker estimates the pose of a set of fiducials with known geometry made of retro-reflective material. It uses a camera assembly

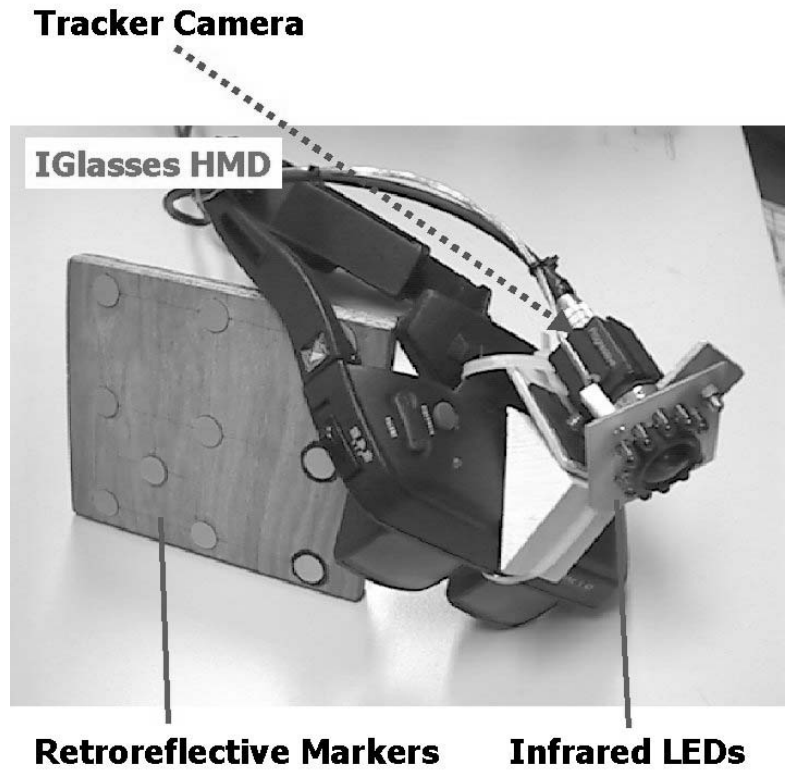

Figure 4. The hardware for the optical seethrough system used in our experiments. A Sony XC-55BB camera with an infrared filter is attached to an off-the-shelf HMD. The camera observes a set of retroreflective markers for tracking.

which is mounted to the HMD. This assembly consists of a CCD camera with an infrared filter and a ring of infrared LEDs mounted around the barrel of the camera. The entire HMD/camera assembly is shown in Figure 4. This tracker uses a technique similar to that described in [27] to estimate the pose of the HMD with respect to the fiducials.

\section{Calibration Requirements}

While working with a bench-top AR system it is important to have a reference coordinate system, in which the locations of the real and virtual objects can be specified. In practice, this coordinate system is in a fixed location such as a table. While the system is running the user's head must be tracked in some way, i.e., with a magnetic tracker or video tracker, so the virtual objects will remain aligned with the real objects as the user moves around. Since this motion is measured in the tracker coordinate system (TCS) and the objects are in the workspace coordinate system (WCS), it is necessary to find a transformation between them so the graphics can be rendered properly.

Figure 5 shows the coordinate systems used in our magnetically tracked setup. The bi-directional arrows 


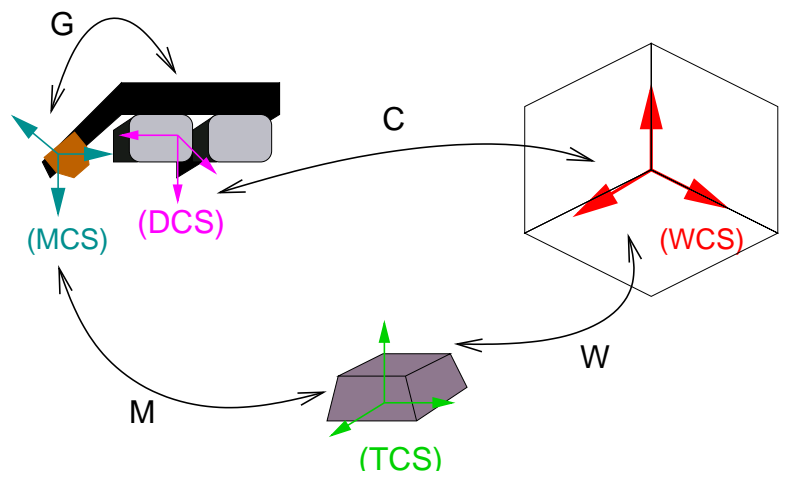

Figure 5. Coordinate transformations in an AR system with a magnetic tracking system.

indicate the coordinate transformations which must be either measured or estimated during system operation. In the figure, $\mathbf{W}$ represents the rigid transformation between the WCS and the TCS. This transformation can be computed by performing a rigid or affine alignment $[13,28]$. Experimental results suggest that the affine method tends to work best since it provides a linear approximation to the aberrations in the magnetic field without resorting to polynomial field adjustment $[11,16,22] . \quad M$ is the transformation from the tracker origin to the marker, which is attached to the HMD. This transformation is measured by the tracking system and reported at each timestep. $\mathbf{G}$ is the transformation from the marker coordinate system to the HMD/eye coordinate system. This transformation is a combination of rigid and projective. It can be estimated using the SPAAM algorithm [29]. During runtime, the transformation we are most interested in is $\mathrm{C}$, which is the transformation used to render the graphics. This transformation is computed by concatenating the former 3 transformations, i.e.,

$$
\mathrm{C}=\mathrm{GMW} .
$$

\section{Evaluating a Calibration}

When designing a scheme to evaluate an AR system, there are many factors to consider. First, the procedure must include the user. Schemes which replace the user with a camera and a rigid mounting fixture do not reflect the situation properly. In fact, much of the error observed in an AR system is from the user. During HMD calibration, for instance, if the user raises their eyebrows, the display moves with respect to the eye and error is introduced into the system.
Another important factor in designing an evaluation system is task complexity. The amount of error a user introduces into the system is reflected in the difficulty of the calibration and evaluation tasks. Several of the users tested remarked that it was difficult to keep the crosshair on the alignment point during the HMD calibration (see [22]). Also, the evaluation task must be designed so it does not give cues to the user, which could influence the results.

A third feature an evaluation scheme must exhibit is accuracy. It must also provide consistent quantitative results from trial to trial. These results must give a clear indication of the accuracy that a user will perceive. It must be able to communicate this accuracy to the user through some feedback mechanism. With this and the two points above in mind, the evaluation scheme can be laid out.

Consider an augmented reality system with an optical see-through HMD and a real-time tracking system. Assuming that the user has calibrated the system along with the HMD, graphics can be overlayed onto the real workspace with some unknown accuracy. The aim is to measure the registration accuracy between the virtual objects and the real objects. The results from these measurements can be used in three different ways. First, calibration accuracy information can be obtained. This information can be used to determine the expected registration accuracy at a given distance. For instance, the system could report, "At $70 \mathrm{~cm}$ away from the target, the system can correctly label items which are $1 \mathrm{~cm}$ apart." This is useful in determining if the calibration is good enough for a particular application. Second, structured errors in the tracking system can be identified and possibly corrected. Structured errors are errors in the system which have a regular form. For instance, problem areas in a magnetic tracker can be found. Third, the structure information may be used to make corrections in the current system calibration to remove these structured errors.

The rest of this section describes a method for evaluating the accuracy of an optical see-through AR system at runtime. The algorithm is given in Section 5.1. Experimental results are presented and discussed in Section 5.2. Section 5.3 discusses the error metric used and its implications. An analysis of the structure of the errors is presented in Section 5.4.

\subsection{Evaluation System and Algorithm}

The evaluation system is comprised of a planar input device and a computer. The input device must be registered into the workspace coordinate system of the AR system being evaluated. For a video-based tracking system, this device may be a graphics tablet with fidu- 


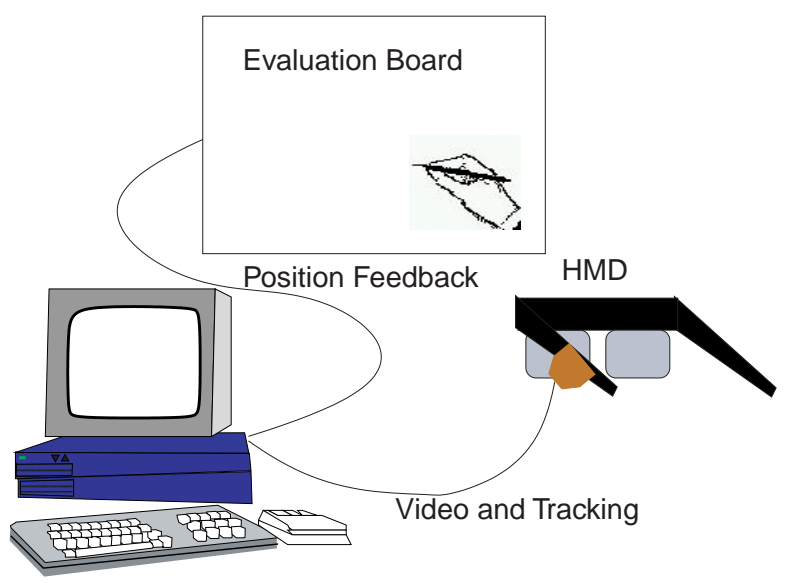

Figure 6. The optical see-through evaluation system.

cials on it so it can be tracked. In the case of a magnetic tracking system, the tracker's stylus can be used. Figure 6 shows a schematic for a system with a video based tracker.

Once the AR system has been calibrated and the input device is registered, the evaluation system can be started. Once started, it will follow the steps shown in Algorithm 1. Note that since the AR system must be running, the user's head is being tracked so they are free to move around during the evaluation process. Also note that the spheres should be displayed in some random order to avoid prompting the user.

\subsection{Experimental Results}

In this section, results from the application of our system to two different AR systems are presented. The first

1. Ask the AR system to superimpose a virtual object, e.g. a small sphere, onto a randomly selected position on the board.

2. Ask the user to touch the board where they perceive the object and record this position.

3. Ask the AR system to render at another location and goto step 1 until the area of the board is evenly covered. Once the area has been covered goto step 4.

4. Compute and display the system error.

Algorithm 1: Evaluation system algorithm. system uses a magnetic tracker; the second uses an infrared video-based tracker. Both systems have monocular displays which were calibrated with the SPAAM method described in [29]. After calibration, the users invoked the evaluation system described in Section 5.1.

We performed 8 experiments using the magnetically tracked system. For each experiment, the user calibrated the HMD and was asked to run the evaluation routine three times over the same grid. The users were asked to confine their heads to 3 rough zones, one zone per pass. The results our system produced follow.

The results from a typical user of the magnetically tracked system are shown in Figure 7. This plot shows the theoretical locations as circles along with their observed locations from three different zones in the workspace. These zones are defined as the 3dimensional regions to which the users' heads were confined when the observations were made. The range of head motion was restricted because the users were seated during the evaluation procedures. Figure 8 shows an example of the three dimensional viewing zones for an evaluation run.

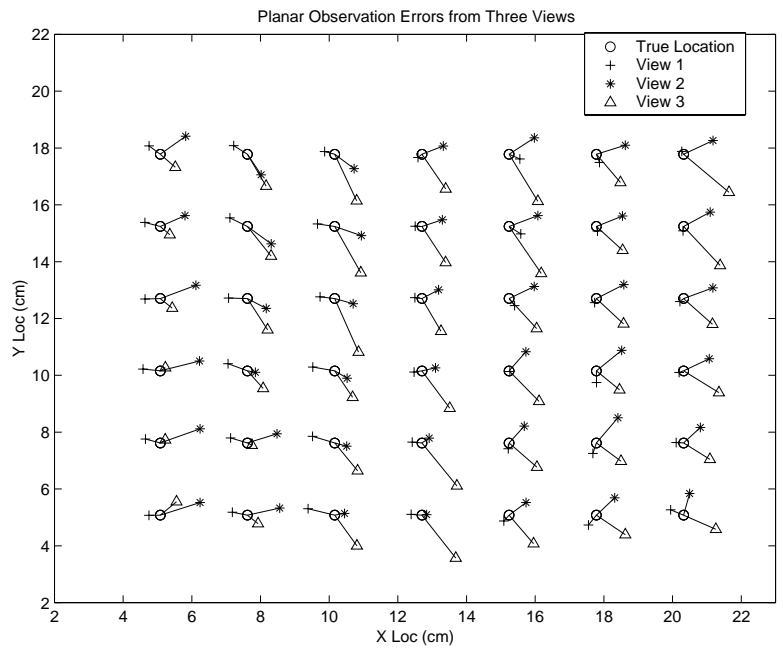

The theoretical points were placed in the plane $z=$ $-0.63 \mathrm{~cm}$. The users recorded observations by clicking the observed location with the stylus from the magnetic tracker. The observations of the points from the three viewing zones are indicated on the plot by pluses, stars and triangles. The mean and maximum errors for each zone and the global errors are tabulated in Table 1. Ta- 


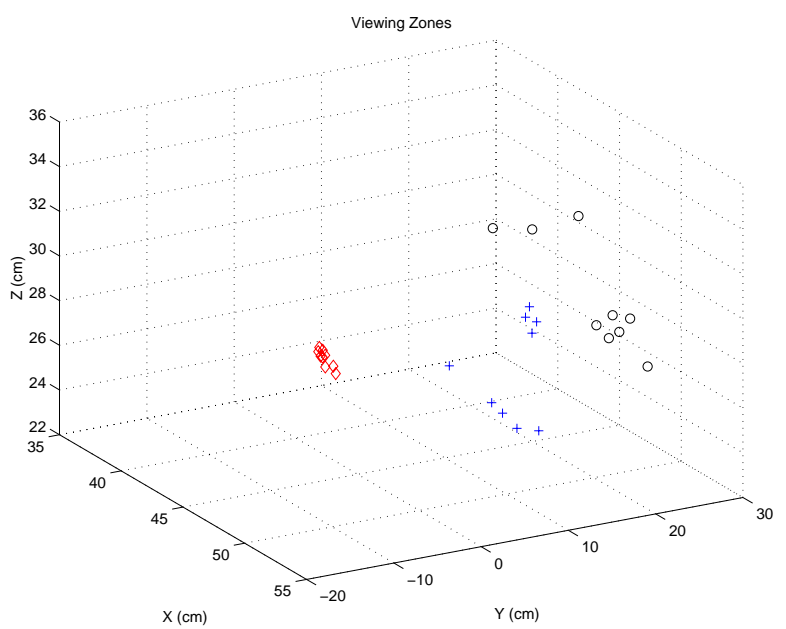

Figure 8. Viewing zones for an evaluation. The points indicate the head positions during the evaluation. The groups of points, i.e., diamonds, circles and pluses represent the zones.

\begin{tabular}{|c|c|c|}
\hline Zone 1 (cm) & Zone 2 (cm) & Zone 3 $(\mathbf{c m})$ \\
\hline 0.7 & 1.1 & 0.3 \\
0.7 & 1.0 & 0.6 \\
0.4 & 0.8 & 1.2 \\
1.2 & 1.5 & 1.1 \\
0.8 & 0.7 & 0.8 \\
1.2 & 1.7 & 0.5 \\
1.2 & 1.6 & 0.8 \\
1.0 & 0.7 & 0.8 \\
\hline
\end{tabular}

Table 2. Average errors for different users from multiple viewing zones (magnetic tracking system.)

\begin{tabular}{|l|c|c|c|}
\hline Observation & Zone 1 $(\mathbf{c m})$ & Zone 2 $(\mathbf{c m})$ & Zone 3 $(\mathbf{c m})$ \\
\hline Mean Error & 0.7 & 1.1 & 0.3 \\
Max Error & 1.2 & 1.7 & 0.5 \\
\hline
\end{tabular}

Table 1. Results for a single user from multiple viewing zones (magnetic tracking system.)

bles 2 and 3 show the mean errors and maximum errors for eight different user sessions. For these eight sessions there were six users. The errors shown in rows 1 and 2 and rows 3 and 4 are from the same users during two different sessions.

From the tables it can be seen that the overall average system accuracy is $0.93 \mathrm{~cm}$. The worst error observed was $4.73 \mathrm{~cm}$, but this was caused by the user moving outside the effective range of the tracker.

We also ran the evaluation system on our video-based tracking setup. Four experiments were run with the same procedure as the magnetic system. Table 4 shows the results from these experiments. A sample plot from one of the experiments is shown in Figure 9. The average error for this system was $0.7 \mathrm{~cm}$. Note that while the video-based system was tested over a larger field, the user was restricted to a single viewing zone to keep the marker in view of the tracker camera at all times.

\begin{tabular}{|c|c|c|}
\hline Zone 1 (cm) & Zone 2 (cm) & Zone 3 (cm) \\
\hline 1.2 & 1.7 & 0.5 \\
0.9 & 1.3 & 0.9 \\
0.8 & 1.3 & 2.0 \\
3.2 & 2.4 & 1.8 \\
1.7 & 1.1 & 1.2 \\
2.3 & 2.8 & 1.2 \\
1.9 & 2.5 & 1.2 \\
4.7 & 3.2 & 3.0 \\
\hline
\end{tabular}

Table 3. Maximum errors for different users from multiple viewing zones (magnetic tracking system.)

\begin{tabular}{|c|c|}
\hline Mean Error $(\mathbf{c m})$ & Max Error $(\mathbf{c m})$ \\
\hline 0.3 & 0.6 \\
0.7 & 1.6 \\
1.1 & 2.1 \\
0.7 & 1.3 \\
\hline
\end{tabular}

Table 4. Mean and maximum errors for different users (video-based tracking system.) 


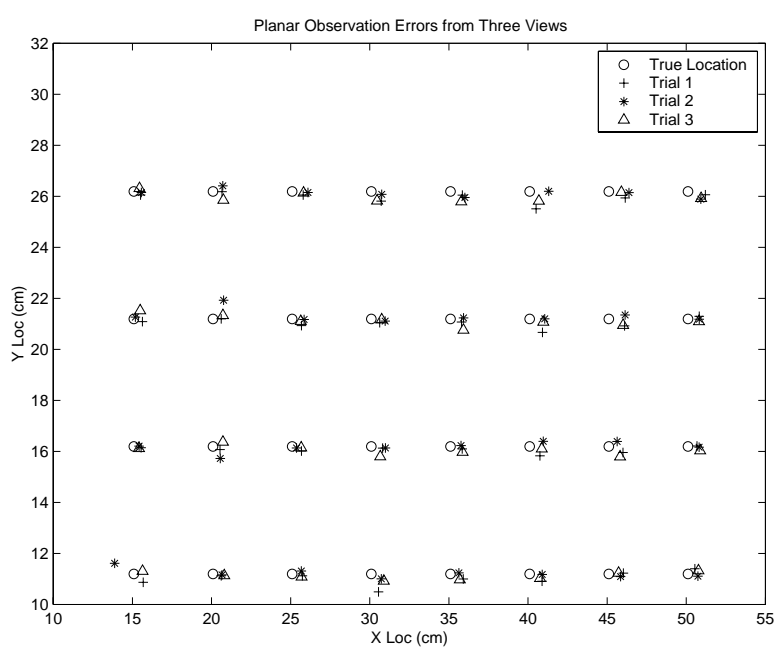

Figure 9. Error fields for an AR system which uses a video-based tracker. These errors are measured in the plane of the evaluation board.

\subsection{Planar Evaluation Analysis}

From the evaluation data, there are three points of interest, $\mathbf{p}_{t}, \mathbf{p}_{o}$, and $\mathbf{p}_{b}$. The first point, $\mathbf{p}_{t}$, is the theoretical location of the virtual point in the workspace coordinate system. The coordinates of this point should be unknown to the user so there is no bias in the results. The theoretical point is rendered in the display using the calibration parameters of the system. This produces the second point, $\mathbf{p}_{o} . \mathbf{p}_{o}$ is the location where the user perceives the point to be in the workspace. In principle, it is difficult for the user to tell the precise 3-dimensional location of the point $\mathbf{p}_{o}$ since we are working with a monocular system. If the HMD and tracker calibrations are exact, then $\mathbf{p}_{t}$ and $\mathbf{p}_{o}$ will coincide. As this is not usually the case, a final point, $\mathbf{p}_{b}$ must be introduced. $\mathbf{p}_{b}$ is the projection of $\mathbf{p}_{o}$ into the plane of the evaluation board. This point is selected by the user when they touch the board during system evaluation. Figure 10 shows the geometry of the 3 points.

It is important to note that the planar error metric completely depends on the user's viewpoint. Figure 11 illustrates this point. The observed error from the viewpoint $E$ is larger than that of $E^{\prime}$. Also notice that from multiple views it is possible to estimate the location of $\mathbf{p}_{o}$ through the use of point reconstruction techniques. This issue is addressed in Section 5.4

Once the data has been gathered, the evaluation system computes the Euclidean distances between each of the theoretical points and their projected observed lo-

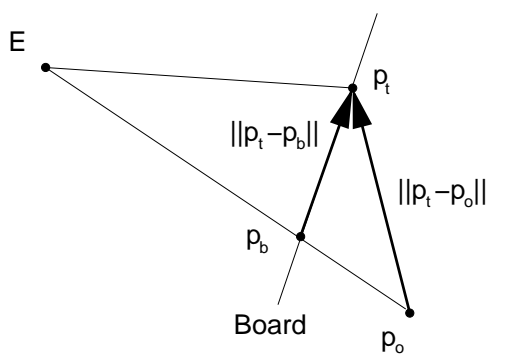

Figure 10. Error is measured in the plane of the evaluation board as seen from the eyepoint, $E$. The true location is $\mathrm{p}_{t}$, the observed location is $\mathrm{p}_{o}$ and the projection of the observed point onto the board is $p_{b}$.

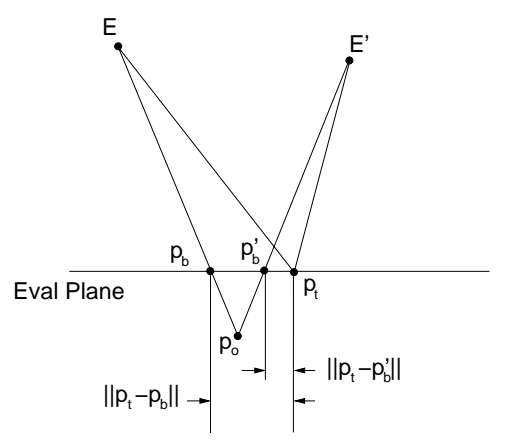

Figure 11. Error in the plane of the evaluation board as seen from the eye-points, $E$ and $E^{\prime}$. The error $\left\|\mathbf{p}_{t}-\mathbf{p}_{b}\right\|$ is larger than $\left\|\mathbf{p}_{t}-\mathbf{p}_{b}^{\prime}\right\|$.

cations, i.e., $\left\|\mathbf{p}_{t}-\mathbf{p}_{b}\right\|$, as shown in Figure 10. The statistics from these computed distances can be used as estimates of tolerances for the current calibration. The errors could also be used to give more direct feedback, such as drawing spheres at each of the picked locations with colors and/or radii corresponding to the amount of error. Additionally, since we are collecting a set of 2D/3D correspondences, the evaluation data can be added to the points used in the AR system calibration to improve the registration.

\subsection{Analysis of 3D Structured Errors}

Given multiple observations of a point from different viewpoints along with its rendering parameters, which need to be passed from the AR system to the evaluation system, it is possible to estimate its 3-dimensional location. If the 3-dimensional locations for all of the 


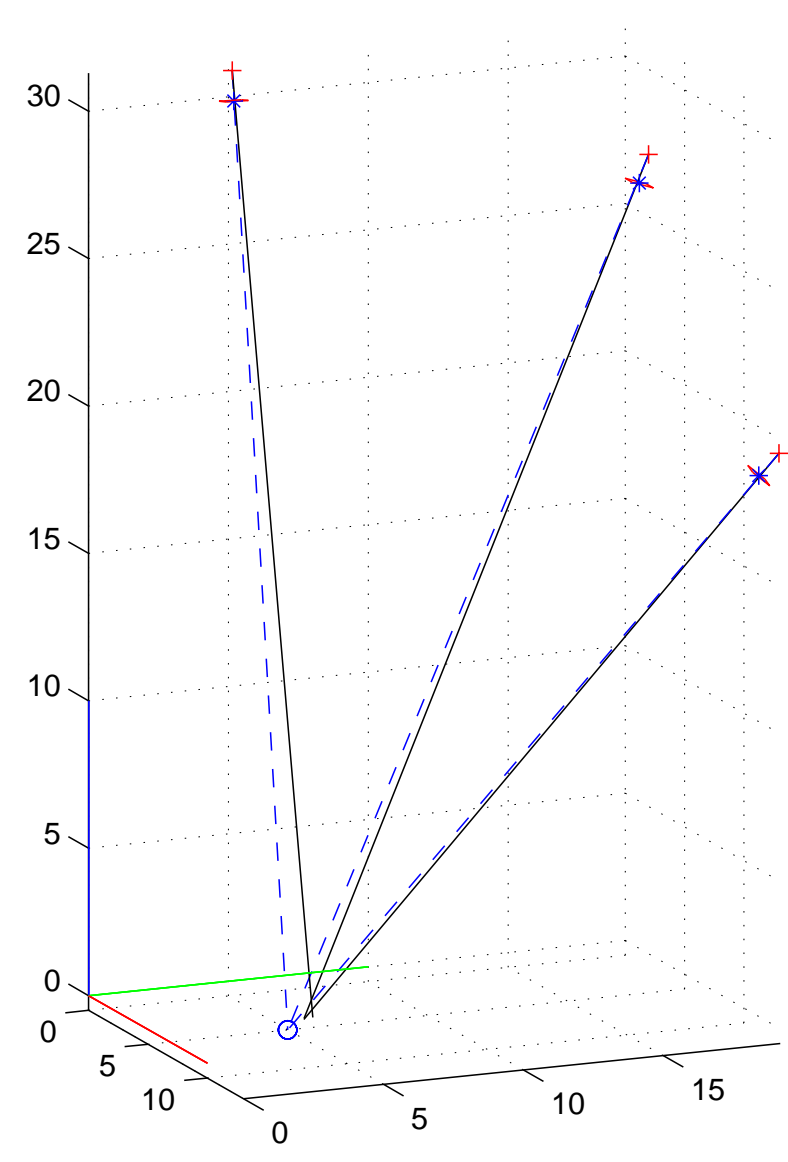

Figure 12. Error simulation environment with structured error plus tracker noise.

points from the evaluation task are estimated they can be looked at collectively as an error field.

Figure 12 depicts an observation error in a simulated case. In this graph, the locations of 3 imaging systems are shown (indicated by the + symbol and the * symbol.) The point that is being rendered is shown as a circle. The observed location in the evaluation plane for each camera lies at the end of the solid rays. In a system with perfect registration and tracking, the solid rays would lie exactly on top of the dashed rays. In practice, there will be positional errors and jitter errors. The positional errors cause the observed location to be different from the theoretical location and the jitter causes the rays of observation to be skew. Using this type of analysis it is possible to characterize the errors in an AR system.

To perform reconstruction, it is first necessary to define the parameters of the system. A point in the workspace coordinate system $\mathbf{M}=[x, y, z, 1]^{T}$ is projected into the image plane at location $\mathbf{m}=[u, v, 1]^{T}$

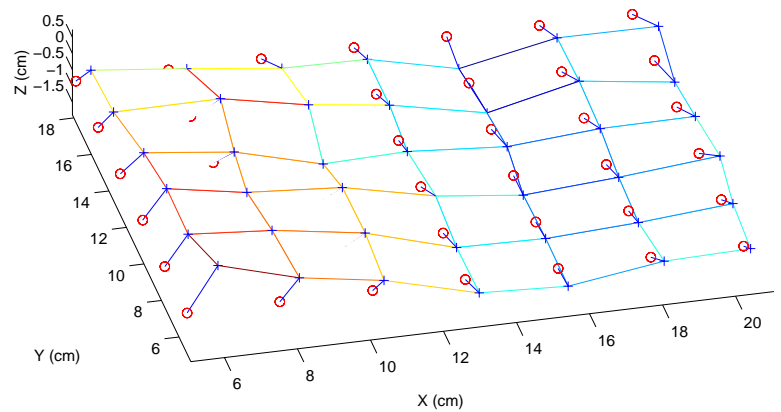

Figure 13. Reconstructed locations using a linear reconstruction method.

with the standard rendering equation, i.e.,

$$
\lambda \mathbf{m}=\mathbf{P M},
$$

where $\mathbf{P}$ is a $3 \times 4$ projection matrix. Using Equation 2 it is possible to solve a linear system to approximate the observed 3-dimensional location of $\mathbf{M}$. This estimation is computed using a standard technique from computer vision known as point reconstruction [5, 10,21].

Figure 13 shows the resulting error field which was reconstructed by this linear technique. In this plot, the circles represent the theoretical locations of the points and the plus signs and mesh represent the reconstructed locations and error field approximation.

From the point locations and the residuals of the reconstruction it is possible to characterize the system errors. If the residuals are very small, but there are significant reconstruction errors, then the error is coming from the system calibration. In this case it should be possible to make a correction to the calibration to compensate for the error. If, however the residuals are very large, then the system is suffering from unstructured tracker noise.

\section{Discussion}

This paper presents a general method to quantitatively evaluate an optical see-through augmented reality system. This system differs from existing methods in three ways. First, it allows a user to measure the error of the system while it is running. Second, it produces quantitative measurement of the error in registration of the real and the virtual. Finally, this error estimate includes the user as part of the system. With this system it is possible to estimate the accuracy limits of an application and detect structured errors in an optical seethrough AR system. 
Several conclusions can be drawn from this work. First, an AR system must be evaluated as a whole. While the errors of individual system components are both useful and meaningful to an implementor, they would be of little value to a maintenance technician using an AR system to perform repairs. Users need concise, simple feedback on the current system accuracy.

Second, during an evaluation of an AR system, the user must be factored in. Since all of the calibration procedures for the system components involve a manual step, there is a possibility that the user can introduce errors into the system. This is especially true if the user is ignorant of actions which may cause errors, such as facial muscle contractions during the calibration, e.g., talking, raising the brow, etc. Simplicity is a must for any calibration algorithm. If the user has to perform some difficult action, it is likely they will inject errors into the system. Other factors may cause errors during calibration and evaluation. For instance, poor lighting which can make alignment of the stylus to the observed point less accurate since the user has difficulty seeing the stylus tip through the darkened display.

Third, since the observed errors are dependent on the viewpoint, the evaluation should be performed from all zones that the user intends to work in. Multiple planes may be evaluated throughout the workspace. From multiple views of the same plane the 3-dimensional error can be estimated, provided that the render matrix is known for each point.

\section{Conclusion}

In this paper we summarized a general method to objectively evaluate an optical see-through augmented reality system. Our evaluation system is easy to use and provides quantitative measurements of the errors in the registration of the real and virtual environments while the user is immersed in the the AR system. The system has been designed so that it may easily be used to evaluate an arbitrary AR system. Our system is capable of estimating the accuracy limits of an application and detecting structured errors in the AR system. Additionally, the measurements can be used to make corrections in the AR system calibrations.

\section{References}

[1] R. Azuma. A survey of augmented reality. Presence: Teleoperators and Virtual Environments, 6(4):355-385, August 1997.

[2] R. Azuma and G. Bishop. Improving static and dynamic registration in an optical see-through display. Computer Graphics, pages 194-204, July 1994.
[3] M. Bajura, H. Fuchs, and Ohbuchi. Merging virtual objects with the real world: Seeing ultrasound imagery within the patient. Computer Graphics, pages 203-210, July 1992.

[4] M. Bajura and U. Neumann. Dynamic registration correction in augmented-reality systems. In Proceedings of the Virtual Reality Annual International Symposium (VRAIS '95), pages 189-196, 1995.

[5] O. Faugeras. Three-dimensional Computer Vision: A Geometric Viewpoint. MIT Press, Cambridge, MA, 1993.

[6] S. Feiner, B. MacIntyre, and D. Seligmann. Knowledgebased augmented reality. Communications of the ACM, 36(2):53-62, 1993.

[7] H. Fuchs and J. P. Rolland. Optical versus video seethrough head-mounted displays in medical visualization. Presence, 9(3):287-309, 2000.

[8] Y. Genc, N. Navab, M. Tuceryan, and E. McGarrity. Evaluation of optical see-through systems. Technical Report SCR-01-TR-688, Siemens Corporate Research, January 2001.

[9] W. Grimson, G. J. Ettinger, S. White, P. Gleason, T. Lozano-Perez, I. W. M. Wells, and R. Kikinis. Evaluating and validating an automated registration system for enhanced reality visualization in surgery. In Proc. of the IEEE Conference on Computer Vision, Virtual Reality and Robotics in Medicine (CVRMed'95), pages 3-12, 1995.

[10] R. I. Hartley and P. Sturm. Triangulation. Computer Vision and Image Understanding, 68(2):146-157, 1997.

[11] R. Holloway. An Analysis of Registration Errors in a See-Through Head-Mounted Display System for Craniofacial Surgery Planning. PhD thesis, University of North Carolina at Chapel Hill, 1994.

[12] R. L. Holloway. Registration error analysis for augmented reality. Presence: Teleoperators and Virtual Environments, 6(4):413-432, August 1997.

[13] R. Jain, R. Kasturi, and B. G. Schunck. Machine Vision. McGraw-Hill, New York, 1995.

[14] A. Janin, D. Mizell, and T. Caudell. Calibration of headmounted displays for augmented reality applications. In Proc. of the Virtual Reality Annual International Symposium (VRAIS'93), pages 246-255, 1993.

[15] H. Kato and M. Billinghurst. Marker tracking and HMD calibration for a video-based augmented reality conferencing system. In Proceedings of the 2nd IEEE and ACM International Workshop on Augmented Reality '99, pages 85-94, San Francisco, CA, October 20-21 1999.

[16] V. Kindratenko. Calibration of electromagnetic tracking devices. Virtual Reality: Research, Development, and Applications, 4:139-150, 1999.

[17] G. J. Klinker, K. H. Ahlers, D. E. Breen, P.-Y. Chevalier, C. Crampton, D. S. Greer, D. Koller, A. Kramer, E. Rose, M. Tuceryan, and R. T. Whitaker. Confluence of computer vision and interactive graphics for augmented reality. Presence: Teleoperators and Virtual Environments, 6(4):433-451, August 1997.

[18] K. N. Kutulakos and J. R. Vallino. Affine object representations for calibration-free augmented reality. In 
Proceedings of the IEEE Virtual Reality Annual International Symposium, 1996.

[19] W. Lorensen, H. Cline, C. Nafis, R. Kikinis, D. Altobelli, and L. Gleason. Enhancing reality in the operating room. In Proceedings of the IEEE Conference on Visualization, pages 410-415, 1993.

[20] B. MacIntyre and E. M. Coelho. Adapting to dynamic registration errors using level of error (loe) filtering. In International Symposium for Augmented Reality, pages 85-88, Munich, Germany, October 2000.

[21] S. Maybank. Theory of Reconstruction from Image Motion. Springer-Verlag, New York, 1993.

[22] E. McGarrity. Evaluation of calibration for optical seethrough augmented reality systems. Master's thesis, Michigan State University, 2001.

[23] E. McGarrity and M. Tuceryan. A method for calibrating see-through head-mounted displays for AR. In 2nd International Workshop on Augmented Reality (IWAR '99), pages 75-84, San Francisco, CA, October 1999.

[24] J. P. Mellor. Real-time camera calibration for enhanced reality visualizations. In Proc. of the IEEE Conference on Computer Vision Virtual Reality and Robotics in Medicine (CVRMed'95), pages 471-475, 1995.

[25] U. Neumann and Y. Cho. A self-tracking augmented reality system. In Proceedings of the ACM Symposium on Virtual Reality and Applications, pages 109-115, 1996.

[26] T. Oishi and S. Tachi. Methods to calibrate projection transformation parameters for see-through headmounted displays. Presence, 5(1):122-135, 1996.

[27] R. Tsai. A versatile camera calibration technique for high-accuracy $3 \mathrm{D}$ machine vision metrology using offthe-shelf TV cameras. IEEE Journal of Robotics and Automation, RA-3(4):323-344, 1987.

[28] M. Tuceryan, D. Greer, R. Whitaker, D. Breen, C. Crampton, E. Rose, and K. Ahlers. Calibration requirements and procedures for a monitor-based augmented reality system. IEEE Transactions on Visualization and Computer Graphics, 1(3):255-273, September 1995.

[29] M. Tuceryan and N. Navab. Single point active alignment method (SPAAM) for optical see-through HMD calibration for AR. In International Symposium for Augmented Reality, pages 149-158, Munich, Germany, October 2000.

[30] M. Uenohara and T. Kanade. Vision-based object registration for real-time image overlay. In Proc. of the IEEE Conference on Computer Vision, Virtual Reality and Robotics in Medicine (CVRMed'95), pages 13-22, 1995. 